\title{
The Choquet Integral Applied to Ranking Therapies in Radiation Cystitis
}

\author{
Elisabeth Rakus-Andersson ${ }^{1}$, and Janusz Frey ${ }^{2}$ \\ ${ }^{1}$ Blekinge Institute of Technology, Department of Mathematics and Science \\ 37179 Karlskrona, Sweden \\ Elisabeth.Andersson@bth.se \\ ${ }^{2}$ Blekinge County Hospital, Department of Surgery and Urology \\ 37185 Karlskrona, Sweden \\ Janusz.Frey@ltblekinge.se
}

\begin{abstract}
We modify the classical fuzzy decision making model by adopting the concept of the Choquet integral as a measure of the therapy utility, when proving different treatments in radiation cystitis. The objective is to rank therapies as a sequence, commencing with the most efficacious remedy.
\end{abstract}

Keywords: Utility matrix, parametric membership functions, weights of importance, utilities of therapies, Choquet integral.

\section{Introduction}

Radiation cystitis is in general rarely occurring, which makes it very difficult to study in a large group of clinical trials. Most available data about radiation cystitis treatment come from a small number of descriptive studies or from expert opinions [2, 4]. As clinical data are considered to have low quality then physicians, who are still facing patients with a disease hugely influencing quality of life, mostly base on their own experience.

We thus want to test fuzzy decision-making model, regarded as a valuable tool, to help in selecting a patient-tailored treatment in radiation cystitis [12].

Theoretical fuzzy decision-making models [14-15], possessing the utility matrix filled with distinct utilities of pairs (decision, object-state), give rise to own trials of successfully accomplished applications concerning the item of medication $[8,12]$. After interpreting pairs (decision, object-state) as (therapy, symptom), we intend to prove decision-making based on the Choquet integral to extract the optimal treatment in radiation cystitis. We wish to confront the results, obtained by the Choquet integral technique, with another model made for the purpose of radiation cystitis in [12].

In Section 2, we recall the basic data inserted into the model of fuzzy decision making. Section 3 provides weights of importance, regarding the symptoms' priorities to retreat. The discussion about the enumeration of utilities is accomplished in Section 4. The concepts of Choquet integrals, playing role of total utilities of therapies, are proved in Section 5, whereas some conclusions are added to Section 6.

adfa, p. 1, 2011.

(C) Springer-Verlag Berlin Heidelberg 2011 


\section{$2 \quad$ Basic Data in Fuzzy Decision Making}

We introduce the notions of a space of states $X=\left\{x_{1}, \ldots, x_{m}\right\}$ and a decision space (a space of alternatives) $A=\left\{a_{1}, \ldots, a_{n}\right\}$. We consider a decision model, in which $n$ alternatives $a_{1}, \ldots, a_{n} \in A$ act as therapies used to treat patients who suffer from radiation cystitis. The therapies should influence $m$ states $x_{1}, \ldots, x_{m} \in X$, identified with $m$ symptoms typical of the morbid unit considered.

When a decision maker applies therapy $a_{i} \in A, i=1, \ldots, n$, to symptom $x_{j} \in X, j=$ $1, \ldots, m$, then a utility of treating $x_{j}$ by $a_{i}$ is determined. In order to sample all distinct utilities, assigned to pairs $\left(a_{i}, x_{j}\right)$, we estimate the total utility $\boldsymbol{U}_{\boldsymbol{a}_{\boldsymbol{i}}}$ as $[12,14-15]$

$$
U_{a_{i}}=\text { aggregation of } u_{i j}, i=1, \ldots, n, j=1, \ldots, m,
$$

in which each element $u_{i j}$ is a utility following from the decision $a_{i}$ with the result $x_{j}$. The most efficacious therapy will be associated with the highest value of $\boldsymbol{U}_{\boldsymbol{a}_{\boldsymbol{i}}}$.

\section{Example 1}

The symptoms, selected as the most decisive for radiation cystitis, are listed as $x_{1}=$ urgency, $x_{2}=$ dysuria, $x_{3}=$ urinary bladder pain, $x_{4}=$ macrohaematuria, $x_{5}=$ urine retention.

The treatments are extracted among $a_{1}=$ alum irrigation, $a_{2}=$ formalin instillation, $a_{3}=$ D-glucosamine, $a_{4}=$ oestrogens, $a_{5}=$ cystodiathermy, $a_{6}=$ interruption of internal illiac arteries, $a_{7}=$ bilateral percutaneous nephrostomy, $a_{8}=$ ileal diversion (with cystectomy), $a_{9}=$ pentoxyfilline and $a_{10}=$ hyperbaric oxygen.

\section{The Importance Weights Assisting Symptoms}

The purpose of this section is to add other factors having impact on the solution of the decision-making model. We wish the model to be furnished with extraction of the most efficacious treatment, provided that the particular emphasis is also concentrated on assigning differing degrees of importance to states-symptoms [8, 12, 14-15].

Let us associate with each symptom $x_{j}, j=1, \ldots, m$, a non negative number that indicates its power or importance in the decision according to the rule: the higher the number is, the more important role of $x_{j}$ 's retreat will be regarded. We assign $w_{1}, \ldots, w_{m}$ as powers-weights to $x_{1}, \ldots, x_{m}$. A procedure for obtaining a ratio scale of importance for a group of $m$ symptoms is developed by the authors as a novelty.

Generally, if we consider $m$ symptoms $X_{j}$ to find importance weights for them, we want to place them in the sequence $X_{1}>X_{2}>\ldots>X_{m}$ in accordance with the expert's opinion. We explain that the symbol ">" stands for "more important than". We wish the sum of all weights $W_{j}$, tied to $X j, j=1, \ldots, m$, to be 1 as to

$$
m \cdot r+(m-1) \cdot r+\cdots+2 \cdot r+1 \cdot r=1,
$$


where $r$ is a quotient depending on $m$. The weights $W_{j}$ constitute a new order of old $w_{j}$, due to the sequence $X_{1}>X_{2}>\ldots>X_{m}$.

Further,

$$
W_{j}=(m-j+1) \cdot r
$$

for $j=1, \ldots, m$.

\section{Example 2}

The physician intends to release the patient from the symptoms with the following priorities: $x_{4}$ - priority $1, x_{3}$ - priority $2, x_{5}=$ priority $3, x_{2}$ - priority 4 and $x_{1}$ - priority 5.

We thus reorder the sequence of the symptoms $x_{j}$ from Ex.1, named $X_{j}$ now, in a new placement

$$
X_{1}=x_{4}>X_{2}=x_{3}>X_{3}=x_{5}>X_{4}=x_{2}>X_{5}=x_{1} .
$$

For $m=5$, due to (2), the equation $5 \cdot r+4 \cdot r+3 \cdot r+2 \cdot r+1 \cdot r=1$ provides $r=0.066$. We get

$W_{1}=(5-1+1) \cdot 0.066=0.33, W_{2}=(5-2+1) \cdot 0.066=0.264$,

$W_{3}=(5-3+1) \cdot 0.066=0.198, W_{4}=(5-4+1) \cdot 0.066=0.132$,

$W_{5}=(5-5+1) \cdot 0.066$.

The common utility $U_{a_{i}}$ of therapy $a_{i}$ is now computed as

$$
U_{a_{i}}=\sum_{j=1}^{m} U_{i j} \cdot W_{j}
$$

where $U_{i j}$ are the utilities of using $a_{i}$ to treat $X_{j}, j=1, \ldots, m$. We emphasize that $U_{i j}$ replace $u_{i j}$, introduced in (1), in compliance with weighed symptom order $X_{1}>X_{2}>\ldots>X_{m}$.

To find a numerical expression for the utility $U_{i j}$, verbally decided by a physician for each pair $\left(a_{i}, X_{j}\right)$, we suggest the analysis of the process following the next session.

\section{Creation of Numerical Expressions for Utilities}

We first want the utility, estimating the remission of symptom $X_{j}$ after treating it by $a_{i}$, $i=1, \ldots, n, j=1, . ., m$, to be verbally expressed in order to facilitate the communication with a professional adviser. We generate a linguistic list named $L=$ "Utility of applying $a_{i}$ to $X_{j}$ ". $L$ becomes stated, e.g., as [12]

"Utility $U_{i j}$ of applying $a_{i}$ to $X_{j}$ " = $\left\{N_{1}=\right.$ "none", $N_{2}=$ "almost none", $N_{3}=$ "very little", $N_{4}=$ "little", $N_{5}=$ "rather little", $N_{6}=$ "moderate", $N_{7}=$ "rather large", $N_{8}=$ "large", $N_{9}=$ "very large", $N_{10}=$ "almost complete", $N_{11}=$ "complete" $\}$.

Each expression $N_{q}, q=1, . ., 11$, will be replaced by a fuzzy set, also named $N_{q}$.

By linking the therapy to the symptom for pair $\left(a_{i}, X_{j}\right)$, a physician selects expression $N_{q}$ from the list due to his experience. It means that $U_{i j}=N_{q}$ in practice. We, in 
turn, should assign a numerical representative to fuzzy set $N_{q}$, assisting each verbal description.

In order not to generate the boundaries of fuzzy sets $N_{q}$ in an ad hoc manner, we suppose that $L=\left\{N_{1}, \ldots, N_{\omega}\right\}$ is a general linguistic list consisting of $\omega$ words. Each word is associated with a fuzzy set. The number $\omega$ is a positive odd integer. Furthermore, let $E$ be the length of a set $R$, designed for all restrictions of the fuzzy sets from $L$, provided that $z \in R$. We now wish to divide the linguistic terms into three groups recognized as a left group, a middle group and a right group. Albeit the trials of generating membership functions with modifiers for linguistic terms were already accomplished [1,6], we propose the authors' own procedure of adopting parametric sfunctions whose derivations can be followed in [9-11].

The membership functions, assigned to the leftmost terms, are prepared by (5) as

$$
\mu_{N_{t}}(z)=\left\{\begin{array}{cccc}
1 & \text { for } & z \leq \frac{E(\omega-1)}{2(\omega+1)} \delta(t), \\
1-2\left(\frac{z-\frac{E(\omega-1)}{2(\omega+1)} \delta(t)}{\frac{E(\omega-1)}{\omega(\omega+1)} \delta(t)}\right)^{2} & \text { for } & \frac{E(\omega-1)}{2(\omega+1)} \delta(t) \leq z \leq \frac{E(\omega-1)}{2 \omega} \delta(t), \\
2\left(\frac{z-\frac{E(\omega-1)(\omega+2)}{2(\omega+1)} \delta(t)}{\frac{E(\omega-1)}{\omega(\omega+1)} \delta(t)}\right)^{2} & \text { for } & \frac{E(\omega-1)}{2 \omega} \delta(t) \leq z \leq \frac{E(\omega-1)(\omega+2)}{2 \omega(\omega+1)} \delta(t), \\
0 & \text { for } & z \geq \frac{E(\omega-1)(\omega+2)}{2 \omega(\omega+1)} \delta(t),
\end{array}\right.
$$

where $\delta(t)=\frac{2 t}{\omega-1}, t=1, \ldots, \frac{\omega-1}{2}$ is a function depending on left function number $t$.

The membership function in the middle is expanded by (6) in the form of

$$
\mu_{\frac{N^{\omega+1}}{2}}(z)=\left\{\begin{array}{lll}
0 & \text { for } \quad z \leq \frac{E(\omega-2)}{2 \omega}, \\
2\left(\frac{\left.z-\frac{E(\omega-2)}{2 \omega}\right)^{2}}{\frac{E}{\omega}}\right)^{2} & \text { for } & \frac{E(\omega-2)}{2 \omega} \leq z \leq \frac{E(\omega-1)}{2 \omega}, \\
1-2\left(\frac{z-\frac{E}{E}}{\frac{E}{\omega}}\right)^{2} & \text { for } & \frac{E(\omega-1)}{2 \omega} \leq z \leq \frac{E}{2}, \\
1-2\left(\frac{z-\frac{E}{2}}{\frac{E}{\omega}}\right)^{2} & \text { for } & \frac{E}{2} \leq z \leq \frac{E(\omega+1)}{2 \omega}, \\
2\left(\frac{\left.\mathrm{z}-\frac{E(\omega+2)}{2 \omega}\right)^{2}}{\frac{E}{\omega}}\right)^{2} & \text { for } & \frac{E(\omega+1)}{2 \omega} \leq z \leq \frac{E(\omega+2)}{2 \omega} \\
0 & \text { for } & z \geq \frac{E(\omega+2)}{2 \omega} .
\end{array}\right.
$$

Finally, the membership functions on the right-hand side can be yielded by (7) as 


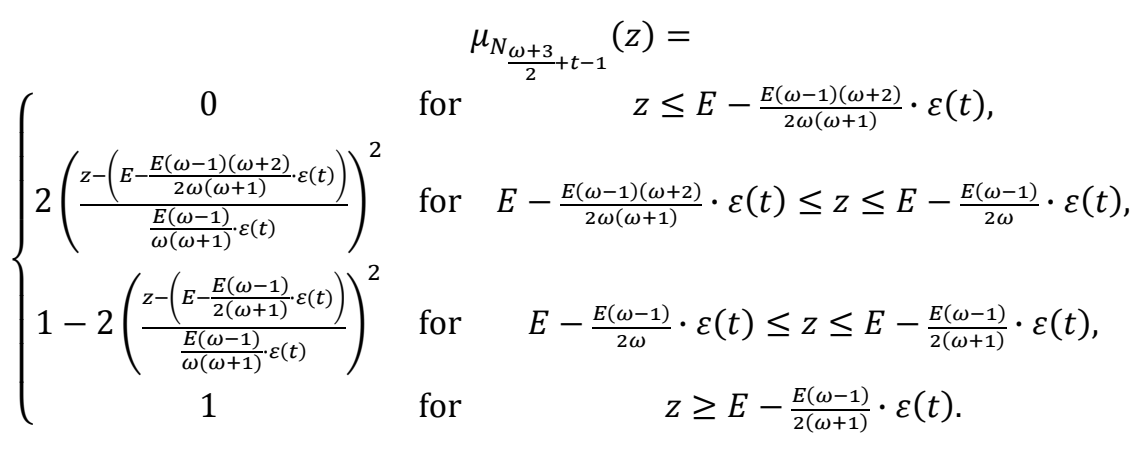

A new function $\varepsilon(t)=1-\frac{2(t-1)}{\omega-1}, t=1, \ldots, \frac{\omega-1}{2}$ allows creating all rightmost functions one by one, when setting $t$-values in (7).

For $\omega=11$ and $E=100$ (the typical length of the reference set $[0,100]$ tested in medical investigations of frequencies) we derive membership functions of $N_{q}, q=$ $1, \ldots, 11$, by employing formulas (5)-(7).

In order to determine the most adequate representative for each $N_{q}, q=1, \ldots, 11$, we first introduce another fuzzy set "total over $[0,100]$ " with the membership function

$$
\mu_{\text {"total over }[0,100] "}(z)=\left\{\begin{array}{cl}
0 & \text { for } \quad z \leq 0 \\
2\left(\frac{z}{100}\right)^{2} & \text { for } 0 \leq z \leq 50, \\
1-\left(\frac{z-100}{100}\right)^{2} & \text { for } 50 \leq z \leq 100 \\
1 & \text { for } \quad z \geq 100
\end{array}\right.
$$

The membership degrees, being the second coordinates of intersection points between membership functions of each $N_{q}$ and "total over [0,100]", will constitute the numerical assignments of the terms allocated in $L$.

Figure 1 collects the graphs of $N_{q}, q=1, \ldots, 11$, and "total over $[0,100]$ ".

We thus connect $N_{1}$ to $0.0054, N_{2}$ to $0.042, N_{3}$ to $0.156, N_{4}$ to $0.274, N_{5}$ to 0.418 and $N_{6}$ to 0.5 . The right fuzzy sets are identified by the following numbers: $N_{7}$ by $0.582, N_{8}$ by $0.726, N_{9}$ by $0.844, N_{10}$ by 0.958 and $N_{11}$ by 0.9946 .

\section{Example 3}

After considering therapies $a_{i}$ and symptoms $X_{j}$ in the radiation cystitis, the judgments of verbal utilities $U_{i j}=N_{q}$ for pairs $\left(a_{i}, X_{j}\right), i=1, \ldots, 10, j=1, \ldots, 5, q=1, \ldots, 11$, are accomplished by physicians. We convert $N_{q}$ into numbers like shown in Table 1. For the reason of sparse data reports, collected for the radiation cystitis, mostly the professional experience of physicians is involved in predicting the utility evaluations [12].

\section{The Choquet Integral as the Total Utility of a Therapy}

The quantities of medical estimates $N_{q}$, taking place in Table 1, are interpreted as utilities $U_{i j}$ of the pairs $\left(a_{i}, X_{j}\right)$. 


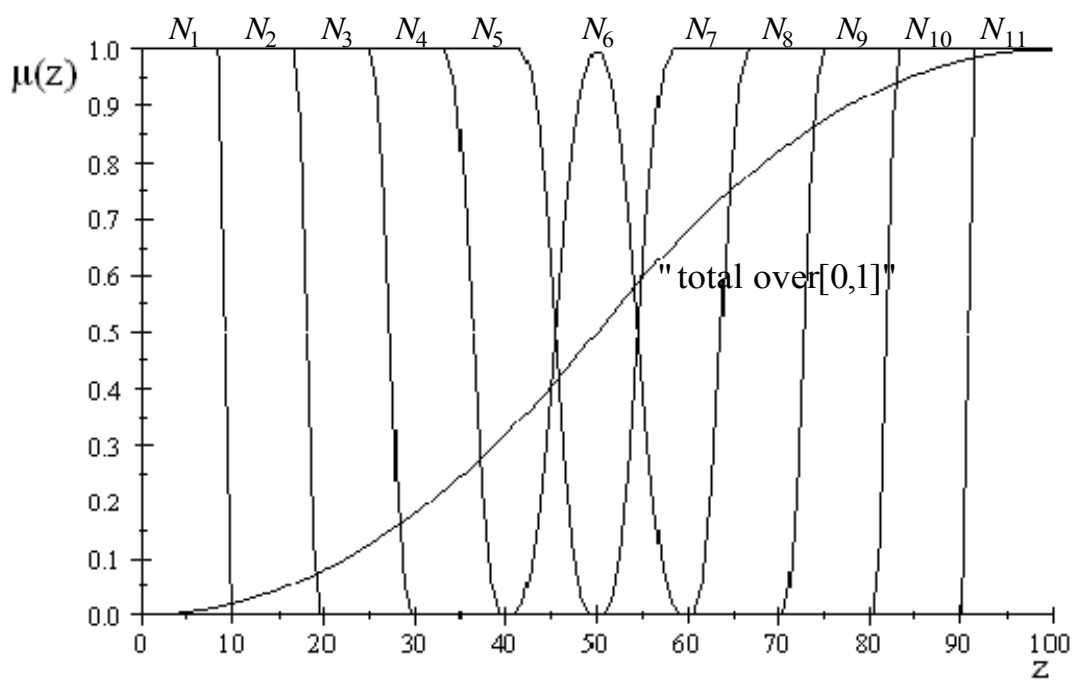

Fig. 1. The fuzzy sets $N_{1}-N_{11}$ and "total over [0, 100]"

Table 1. Utilities $U_{i j}=N_{q}$ of pairs $\left(a_{i}, X_{j}\right)$

\begin{tabular}{l|lllll}
\hline$a_{i} \backslash x_{j}$ & $X_{1}$ & $X_{2}$ & $X_{3}$ & $X_{4}$ & $X_{5}$ \\
\hline$a_{1}$ & $N_{7}=0.582$ & $N_{3}=0.156$ & $N_{5}=0.418$ & $N_{3}=0.156$ & $N_{1}=0.0054$ \\
$a_{2}$ & $N_{8}=0.726$ & $N_{2}=0.042$ & $N_{3}=0.156$ & $N_{2}=0.042$ & $N_{1}=0.0054$ \\
$a_{3}$ & $N_{2}=0.042$ & $N_{3}=0.156$ & $N_{2}=0.042$ & $N_{3}=0.156$ & $N_{3}=0.156$ \\
$a_{4}$ & $N_{5}=0.418$ & $N_{4}=0.274$ & $N_{4}=0.274$ & $N_{6}=0.5$ & $N_{6}=0.5$ \\
$a_{5}$ & $N_{5}=0.418$ & $N_{3}=0.156$ & $N_{5}=0.418$ & $N_{1}=0.0054$ & $N_{1}=0.0054$ \\
$a_{6}$ & $N_{10}=0.958$ & $N_{4}=0.274$ & $N_{3}=0.156$ & $N_{2}=0.042$ & $N_{1}=0.0054$ \\
$a_{7}$ & $N_{6}=0.5$ & $N_{3}=0.156$ & $N_{10}=0.958$ & $N_{8}=0.726$ & $N_{9}=0.844$ \\
$a_{8}$ & $N_{10}=0.958$ & $N_{11}=0.9946$ & $N_{11}=0.9946$ & $N_{11}=0.9946$ & $N_{11}=0.9946$ \\
$a_{9}$ & $N_{7}=0.582$ & $N_{3}=0.156$ & $N_{2}=0.042$ & $N_{4}=0.274$ & $N_{3}=0.156$ \\
$a_{10}$ & $N_{7}=0.582$ & $N_{6}=0.5$ & $N_{7}=0.582$ & $N_{6}=0.5$ & $N_{4}=0.274$ \\
\hline
\end{tabular}

We remember that symptoms $X_{1}, \ldots, X_{m} \in X$ act as objects in $X$. To them let us assign the measures $M\left(X_{j} \mid a_{i}\right)=U_{i j}$, where symbols $X_{j} \mid a_{i}$ reflect the association between symptom $X_{j}$ and medicine $a_{i}, i=1, \ldots, n, j=1, \ldots, m$.

The weights $W_{j}$ are set as the range values $f\left(X_{j}\right)$ of a function $f: X \rightarrow=[0,1]$. In this context formula (4) gets a new shape of

$$
U_{a_{i}}=\sum_{j=1}^{m} U_{i j} \cdot W_{j}=\sum_{j=1}^{m} M\left(X_{j} \mid a_{i}\right) \cdot W_{j}
$$


The second part of formula (9) is comparable to the area of a figure, composed of rectangles. These possess one side equal to $M\left(X_{j} \mid a_{i}\right)$ and the other side measured as the $W_{j}$ value.

\section{Example 4}

Let us estimate the influence of therapy $a_{1}$ on symptoms $X_{j}, j=1, \ldots, 5$, characteristic of the radiation cystitis. The total utility of $a_{1}$ is measured as the surface of the pattern sketched in Fig. 2.

Hence,

$U_{a_{1}}=\sum_{j=1}^{5} M\left(X_{j}\left\lceil a_{1}\right) \cdot W_{j}=0.582 \cdot 0.33+0.156 \cdot 0.264+0.418 \cdot 0.198+\right.$ $0.156 \cdot 0.132+0.0054 \cdot 0.066=0.337$.

The same result will be reached after converting the contents of Fig. 2 to the figure drawn in Fig. 3.

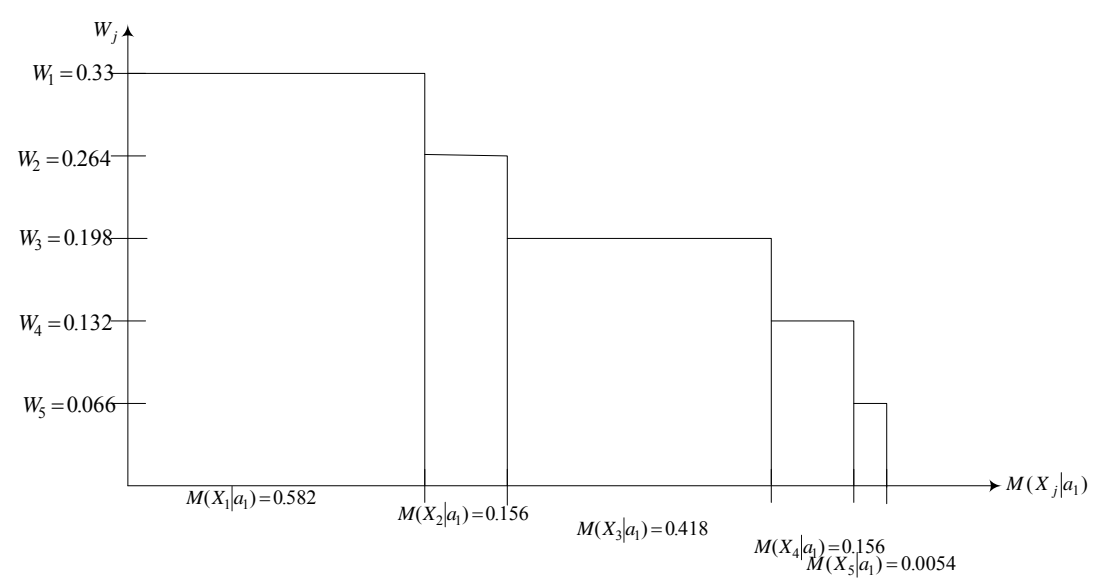

Fig. 2. The graph of $U_{a_{1}}=\sum_{j=1}^{5} M\left(X_{j}\left[a_{1}\right) \cdot W_{j}\right.$

The area of the pattern, sketched in Fig. 3, corresponds to

$U_{a_{1}}=0.582(0.33-0.264)+(0.582+0.156)(0.264-0.198)+(0.582+$ $0.156+0.418)(0.198-0.132)+(0.582+0.156+0.418+0.156)(0.132-$ $0.066)+(0.582+0.156+0.418+0.156+0.0054)(0.066-0)=0.337$.

The last result in Ex. 4 is fully compatible with

$$
\begin{aligned}
& U_{a_{1}}= \\
& \left(W_{1}-W_{2}\right) \cdot M\left(X_{1 \leq s \leq j} \mid a_{1}: f\left(X_{1 \leq s \leq j}\right) \geq W_{1}\right)_{j=1}+ \\
& \left(W_{2}-W_{3}\right) \cdot M\left(X_{1 \leq s \leq j} \mid a_{1}: f\left(X_{1 \leq s \leq j}\right) \geq W_{2}\right)_{j=2}{ }^{+} \\
& \left(W_{3}-W_{4}\right) \cdot M\left(X_{1 \leq s \leq j} \mid a_{1}: f\left(X_{1 \leq s \leq j}\right) \geq W_{3}\right)_{j=3}+
\end{aligned}
$$




$$
\begin{aligned}
& \left(W_{4}-W_{5}\right) \cdot M\left(X_{1 \leq s \leq j} \mid a_{1}: f\left(X_{1 \leq s \leq j}\right) \geq W_{4}\right)_{j=4}+ \\
& \left(W_{5}-W_{6}\right) \cdot M\left(X_{1 \leq s \leq j} \mid a_{1}: f\left(X_{1 \leq s \leq j}\right) \geq W_{5}\right)_{j=5} \\
& =\sum_{j=1}^{5}\left(W_{j}-W_{j+1}\right) \cdot M\left(X_{1 \leq s \leq j} \mid a_{1}\right): f\left(X_{1 \leq s \leq j}\right) \geq W_{j}
\end{aligned}
$$

provided that $W_{6}=0$ and $M\left(X_{1 \leq s \leq j} \mid a_{1}\right)=\sum_{s=1}^{j} M\left(X_{s} \mid a_{1}\right)$.

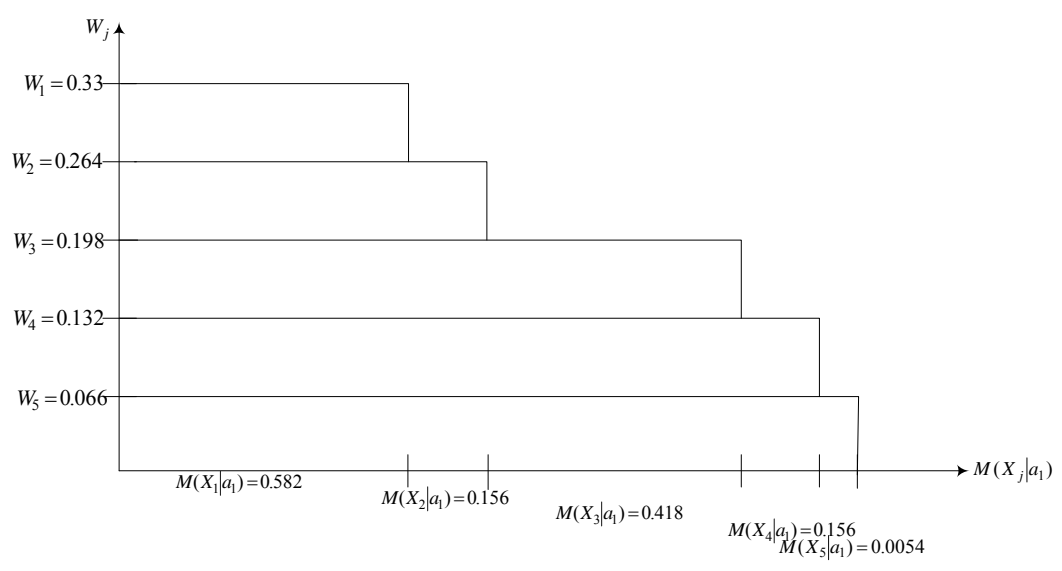

Fig. 3. The pattern of computing $U_{a_{1}}$ after converting Fig. 2

Formula (10), on the other hand, can be formalized as

$$
\sum_{j=1}^{5}\left(W_{j}-W_{j+1}\right) \cdot M\left(X_{1 \leq s \leq j} \mid a_{1}\right): f\left(X_{1 \leq s \leq j}\right) \geq W_{j}=\int_{X_{j} \in\left\{X_{1}, X_{2}, X_{3}, X_{4}, X_{5}\right\}} f\left(X_{j}\right) d M\left(X_{j} \mid a_{1}\right)
$$

and, after the confrontation with [3,5,13], is identified as the Choquet integral over $X$ $=\left\{X_{1}, X_{2}, X_{3}, X_{4}, X_{5}\right\}$. In general, we are able to perform the computations of total utilities of $a_{i}, i=1, \ldots, n$, by [7]

$U_{a_{i}}=\int_{X_{j} \in X} f\left(X_{j}\right) d M\left(\left(X_{j} \mid a_{i}\right)=\sum_{j=1}^{m}\left(W_{j}-W_{j+1}\right) \cdot M\left(X_{1 \leq s \leq j} \mid a_{1}\right): f\left(X_{1 \leq s \leq j}\right) \geq W_{j}\right.$

We perform (12) to estimate: $U_{a_{2}}=0.287, U_{a_{3}}=0.094, U_{a_{4}}=0.366, U_{a_{5}}=0.263$, $U_{a_{6}}=0.425, U_{a_{7}}=0.547, U_{a_{8}}=0.973, U_{a_{9}}=0.288$, and $U_{a_{10}}=0.523$. 
As the most efficacious therapy, $a_{i}$ assists the largest $U_{a_{i}}$ value, $i=1, \ldots, 10$. We will thus set the therapies in the sequence $a_{8}>a_{7}>a_{10}>a_{6}>a_{4}>a_{1}>$ $a_{9}>a_{2}>a_{5}>a_{3}$, when assuming that " $>$ " means " $a_{i}$ shows the stronger power in receding symptoms than $a_{c}, i, c=1, \ldots, n$ ".

\section{Conclusions}

The basis of investigations has been mostly restricted to a judgment of the therapy influence on clinical symptoms characterizing radiation cystitis. In the classical model of fuzzy decision making [14-15] we have also employed the indices of the symptoms' importance to emphasize the essence of the symptom priority to recede in the final decision. By interpreting the utilities of treatments as measures we have furnished total utility estimates with such tools as the Choquet integrals to extend the model of the classical fuzzy decision-making. This complement to decision making constitutes an original contribution as it has been impossible to find the similar effects in literature apart from our own [7]. The method of constructing families of membership functions, depending only of the length of a reference set and the number of constraints, reveals another own idea of the authors.

The results of the decision model, obtained in this paper, converge to the final decision from [12]. These seem to be reasonable from the clinicians' point of view and, with some exceptions, they match results of the algorithm proposed by MartinezRodriques [4]. Nevertheless, we would strongly emphasize that the symptoms, their intensities and treatment efficacies are mostly based on the personal experience and obviously can vary among the centers. We also note that the treatment of radiation cystitis is most often multimodal when combining various methods. The final scale of therapy priorities from Section 5 should absolutely not be regarded as a guideline for future prognoses of treatments but the model itself, with dynamic input categories, seems to be a very valuable tool helping to determine the appropriate treatment path.

To sum up we would like to state that computational intelligence methods can constitute perfect bridges between the expert judgments and real evidence based medicine in case of diseases that lack data from the good quality clinical trials.

\section{References}

1. Bouchon-Meunier, B, Jia, Y: Linguistic modifiers and imprecise categories. Special Issue: Uncertanty Management in Knowledge-based Systems 7(1), 25-36 (1992)

2. Denton, A.S., Clarke, N., Maher, J.: Non-surgical Interventions for Late Radiation Cystitis in Patients who Have Received Radical Radiotherapy to the Pelvis. Wiley Online The Cochrane Library, http:// onlinelibrary.wiley.com/doi/ 10.1002/ 14651858.CD 001 773/ pdf (2009)

3. Grabisch, M., Murofushi, T., Sugeno, M., Kacprzyk, J.: Fuzzy Measures and Integrals. Theory and Applications. Physica Verlag, Berlin Heidelberg (2000)

4. Martinez-Rodrigues, R., Areal Calama, J., Buisan Rueda, O., González Satue, C., Sanchez Macias, J., Arzoz Fabregas, M., Gago Ramos, J., Bayona Arenas, S., Ibarz Servio, L., 
Saladié Roig, J.M.: Practical Treatment Approach of Radiation Induced Cystitis. Actas Urol Esp. 34(7), 603-609 (2010)

5. Mirofushi, T., Sugeno, M.: An Interpretation of Fuzzy Measures and the Choquet Integral as an Integral with Respect to a Fuzzy Measure. Fuzzy Sets and Systems 29, 201-227 (1989)

6. Novák, V., Perfilieva, I.: Evaluating of Linguistic Expressions and Functional Fuzzy Theories in Fuzzy Logic. In: Zadeh, L. A., Kacprzyk, J. (eds.): Computing with Words in Information - Intelligent Systems, Springer-verlag, Berlin Heidelberg New York, 383-406 (1999)

7. Rakus-Andersson, E., Jogreus C.: The Choquet and Sugeno Integrals as Measures of Total Effectiveness of Medicines. In: Castillo, O., Melin, P., Ross, O. M., Cruz, M., Pedrycz, W., Kacprzyk, J. (Eds):Theoretical Advances and Applications of Fuzzy Logic and Soft Computing, Springer-verlag, 253-262 (2007)

8. Rakus-Andersson, E.: Decision-making Techniques in Ranking of Medicine Effectiveness. In: Sordo, M., Vaidya, W., Jain, L. C. (Eds): Advanced Computational Intelligence Paradigms in Healthcare 3, Springer-verlag, Berlin Heidelberg, 51-73 (2008)

9. Rakus-Andersson, E.: Adjusted s-parametric Functions in the Creation of Symmetric Constraints. Proceedings of the $10^{\text {th }}$ International Conference on Intelligent Systems Design and Applications - ISDA 2010, 451-456 (2010)

10. Rakus-Andersson, E.: The Mamdani Controller with Modeled Families of Constraints in Evaluation of Cancer Patient Survival Length. In: Ramanna, S., Jain, L., Howlett, R. (Eds): Emerging Paradigms in Machine Learning, Springer, Berlin Heidelberg New York, 359-378 (2012)

11. Rakus-Andersson, E.: Selected Algorithms of Computational Intelligence in Surgery Decision Making. Open Access book Gastroenterology in SCITECH http://www.intechopen.com/articles/show/title/selected-algorithms-of-computationalintelligence-in-cancer-surgery-decision-making (2012)

12. Rakus-Andersson, E, Frey, J.: $\alpha$-cut Fuzzy Numbers as Utilities of Decision Making in Treatment of Radiation Cystitis. Proceedings of the 14th International Conference in Information Processing and Management of Uncertainty in Knowledge-based Systems IPMU 2012, Springer verlag, 140-149 (2012)

13. Sugeno, M.: Fuzzy Measures and Fuzzy Integrals - a Survey. In: Gupta, M. M., Saridis, G. N., Gaines, B. R. (eds.): Fuzzy Automata and Decision Processes, New York NorthHolland, 89-102 (1977)

14. Yager, R. R.: Fuzzy Decision Making Including Unequal Objectives. Fuzzy Sets and Systems 1, 87-95 (1978)

15. Yager, R. R.: Generalized OWA Aggregation Operators. Fuzzy Optimization and Decision Making 3, 93-107 (2004) 